\title{
Experimental characterization of intensity scintillation in the LEO downlink
}

\author{
Analysis of measurements during the KIODO campaign
}

\author{
Florian Moll \\ Institute of Communications and Navigation \\ German Aerospace Center \\ 82234 Wessling, Germany \\ florian.moll@dlr.de
}

\begin{abstract}
- the paper addresses the experimental investigation of intensity scintillation for characterization of optical Low Earth Orbit-ground communications. In the framework of a former measurement campaign in $\mathbf{2 0 0 6}$ and 2009, recordings of intensity scintillation were taken using a pupil plane camera. These recordings are analyzed and intensity scintillation index and correlation width are derived for seven satellite passes. Mean values of the measurements are created and compared to common theory. The mean of correlation width coincides well with the theoretical model. The intensity scintillation index shows deviation in the focusing regime but agreement otherwise.
\end{abstract}

Keywords-free-space optical communications; LEO-downlink; intensity scintillation; measurement analysis; scintillation index; correlation width

\section{INTRODUCTION}

Future satellite missions will employ ever more powerful sensors that produce higher and higher data volumes. The used data link has to supply the capacity that can cope with the ever increasing demands. Free-space optical communications are the technology that can meet the requirements. This paper treats the scenario of Low Earth Orbit (LEO) to ground links. The transmitter is an optical terminal installed on the LEO satellite. The receiver is a ground station at an arbitrary place, in the case of this study located near Munich, Germany. For an optimized design of transmitter and receiver, thorough knowledge of the communication channel is necessary. The current analysis treats the effect of atmospheric turbulence on the laser beam, in particular intensity scintillation. Various models do exist that describe and may predict behaviour of atmospheric turbulence and can be found in common literature, e.g. [1][2][3]. Turbulence effects in the LEO-ground scenario are described in particular in [4]. In the current paper, a preliminary analysis of scintillation measurements is presented that were derived from recorded pupil plane images. The receiver was the Optical Ground Station Oberpfaffenhofen near Munich, Germany. The measures of scintillation are the scintillation index and correlation width of the received intensity field. The measurements were undertaken in the framework of the KIODO (KIrari Optical Downlink to Oberpfaffenhofen) campaigns in 2006 and 2009. The laser terminal on board the OICETS (Optical Inter-orbit
Communications Engineering Test Satellite) satellite acted as laser source [5][6]. The motivation of this analysis is the future development of a channel model that bases on common theory of scintillation and direct scintillation measurements.

\section{THEORY}

The scintillation index of intensity fluctuations is defined as

$\sigma_{\mathrm{I}}^{2}=\frac{\left\langle I^{2}-\langle I\rangle^{2}\right\rangle}{\langle I\rangle^{2}}$

with $I\left[\mathrm{~W} / \mathrm{m}^{2}\right]$ being the intensity at the plane of observation which is usually the entrance pupil of the measurement system. For the LEO-ground scenario, it is assumed that the e.-m. wave is accurately modelled by a plane wave. In this case, the scintillation index for weak, moderate and strong fluctuations assuming a Kolmogorov turbulence spectrum is given by [2]

$$
\sigma_{\mathrm{I}}^{2}=\exp \left[\frac{0.49 \sigma_{\mathrm{R}}^{2}}{\left(1+1.11 \sigma_{R}^{12 / 5}\right)^{7 / 6}}+\frac{0.51 \sigma_{\mathrm{R}}^{2}}{\left(1+0.69 \sigma_{R}^{12 / 5}\right)^{5 / 6}}\right]-1
$$

with the Rytov index $\sigma_{\mathrm{R}}{ }^{2}[-]$ for a slant path given by

$$
\sigma_{\mathrm{R}}^{2}=\sigma_{\mathrm{I}, \mathrm{wf}}^{2}=2.25 k^{7 / 6} \sec ^{11 / 6}(\zeta) \int_{h_{0}}^{H} C_{\mathrm{n}}^{2}(h)\left(h-h_{0}\right)^{5 / 6} d h
$$

The Rytov index is equivalent to the scintillation index $\sigma_{\mathrm{I}}^{2}[-]$ of a plane wave in weak fluctuations conditions. The variable $h[\mathrm{~m}]$ is the control variable in vertical direction, $k\left[\mathrm{~m}^{-1}\right]$ is the wavenumber, $C_{\mathrm{n}}^{2}\left[\mathrm{~m}^{-2 / 3}\right]$ is the vertical index of refraction structure constant profile, $\zeta[\mathrm{deg}]$ is the zenith angle, $H[\mathrm{~m}]$ is the top of the atmosphere and $h_{0}[\mathrm{~m}]$ is the height of the receiver. The covariance function of intensity $B_{\mathrm{I}}(\rho)$ is given by [2]

$$
\begin{gathered}
B_{\mathrm{I}}(\rho)=\exp \left[\frac{\mu_{4 \mathrm{~d}}(\rho)}{\mu_{4 \mathrm{~d}}(0)} \sigma_{\ln \mathrm{X}}^{2}+0.99\left(\frac{k \rho^{2} \eta_{\mathrm{Y}}}{L}\right)^{5 / 12}\right. \\
\left.K_{5 / 6}\left(\sqrt{\frac{k \rho^{2} \eta_{\mathrm{Y}}}{L}}\right) \sigma_{\ln \mathrm{Y}}^{2}\right]-1
\end{gathered}
$$




$$
\begin{gathered}
\mu_{4 \mathrm{~d}}(\rho)=\int_{h_{0}}^{H} \frac{C_{\mathrm{n}}^{2}(h)}{\xi^{1 / 3}(1-5 / 8 \xi)^{7 / 5}} . \\
{ }_{1} F_{1}\left[\frac{7}{5} ; 1 ; \frac{-k \rho^{2} \eta_{\mathrm{X}}}{8 L \xi^{5 / 3}(1-5 / 8 \xi)}\right] d h
\end{gathered}
$$

$K_{\mathrm{v}}(\mathrm{x})$ is a modified Bessel function, ${ }_{1} F_{1}(\mathrm{a} ; \mathrm{b} ; \mathrm{c})$ is the Hypergeometric function, $L[\mathrm{~m}]$ is the distance between transmitter and receiver. The parameters $\eta_{\mathrm{X}}, \eta_{\mathrm{Y}}, \sigma_{\ln \mathrm{X}}{ }^{2}$ and $\sigma_{\ln \mathrm{Y}}{ }^{2}$ are given by

$$
\begin{aligned}
& \eta_{\mathrm{X}}=\frac{0.92}{1+1.11 \sigma_{\mathrm{R}}^{12 / 5}}, \\
& \eta_{\mathrm{Y}}=3\left(1+0.69 \sigma_{\mathrm{R}}^{12 / 5}\right), \\
& \sigma_{\ln \mathrm{X}}^{2}=\frac{0.49 \sigma_{\mathrm{R}}^{2}}{\left(1+1.11 \sigma_{\mathrm{R}}^{12 / 5}\right)},
\end{aligned}
$$

and

$$
\sigma_{\ln Y}^{2}=\frac{0.51 \sigma_{\mathrm{R}}^{2}}{\left(1+0.69 \sigma_{\mathrm{R}}^{12 / 5}\right)} .
$$

The deviation of scintillation index derived from the weak fluctuation model $\left(\sigma_{\mathrm{I}, \mathrm{wf}}^{2}\right)$, equation (3), and the strong fluctuation model, equation (2), comes clear in Fig. 1. The point where the blue and the black curves start to deviate is considered to be the rise of saturation. The peak of scintillation is at about 15 to $20 \mathrm{deg}$ with $\sigma_{\mathrm{I}}^{2}=1.2$. Inner and outer scale effects are neglected in this calculation since a Kolmogorov spectrum is assumed. Therefore, the scintillation index in the saturation regime is expected to be underestimated here and actually higher in a real scenario.

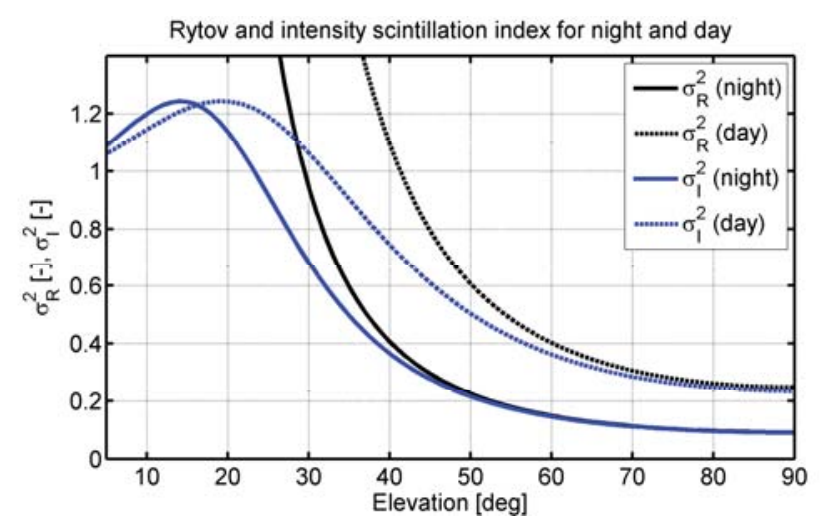

Fig. 1. Rytov index and scintillation index over elevation for two different ground values of $C_{\mathrm{n}}^{2}$ representing daytime $\left(\mathrm{C}_{\mathrm{n}}^{2}=1.7 \mathrm{e}-13 \mathrm{~m}^{-2 / 3}\right)$ and nighttime $\left(\mathrm{C}_{\mathrm{n}}^{2}=1.7 \mathrm{e}-14 \mathrm{~m}^{-2 / 3}\right)$ conditions.

\section{MEASUREMENT SETUP}

The measurements are obtained by use of DLR's Optical Ground Station Oberpfaffenhofen within two measurement campaigns: KIODO 2006 and 2009. The main specifications are given in Table I. AND 2009

\begin{tabular}{|l|c|c|}
\hline Specification & KIODO 2006 & KIODO 2009 \\
\hline Wavelength & $847 \mathrm{~nm}$ & $847 \mathrm{~nm}$ \\
\hline Sensor type & $\mathrm{Si}$ & $\mathrm{Si}$ \\
\hline Resolution & $696 \times 520$ & $1392 \times 1040$ \\
\hline Pixel pitch & $13.7 \mu \mathrm{m}$ & $6.45 \mu \mathrm{m}$ \\
\hline Pupil size & $5.2 \mathrm{~mm}$ & $5.2 \mathrm{~mm}$ \\
\hline Magnification & 77 & 77 \\
\hline Frame rate & $15 \mathrm{fps}$ & $10 \mathrm{fps}$ \\
\hline
\end{tabular}

An illustration of the optical systems function is given in Fig. 2. The signal is received and the beam compressed by an afocal system (40 cm Cassegrain telescope and collimator lens), split by several beam splitter cubes (BSCs) and distributed to the installed measurement devices. The device of interest here is the pupil camera which utilizes an additional imaging system, i.e. the presented analysis is based on a pupil plane imager. Each pixel of the camera can be seen as an array of point receivers with uniform separation (pixel pitch). The ex-aperture size of one pixel is determined by the pixel pitch and the magnification, resulting in a spatial sampling of $1.1 \mathrm{~mm}$ (KIODO 2006) and $0.5 \mathrm{~mm}$ (KIODO 2009). Since these sizes are much smaller than the typical size of the intensity correlation width, no aperture averaging takes place. The tracking camera is devoted to the optical tracking system of the ground station which guarantees link lock during the satellite pass.

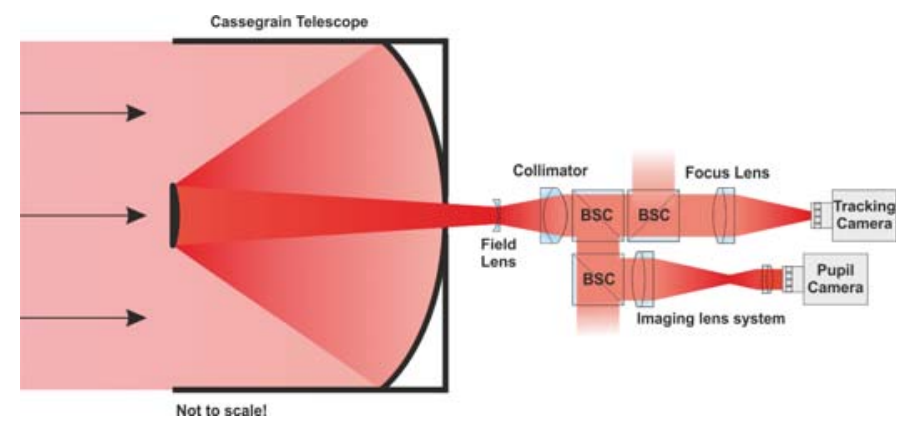

Fig. 2. Concept of optical setup for measurement of field intensity in pupil plane.

\section{ANALYSIS METHOD}

Pre-processing of the images is performed involving two steps: First, invalid images are identified and filtered out by manual inspection and evaluation of signal quality to obtain the measurement images $I_{\text {meas }}(x, y)$. The variables $x$ and $y$ denote the image pixel indices in the two directions. Second, the camera offset is subtracted and a mask image $M(x, y)$ and scaling image $S(x, y)$ are created. The mask defines the area of interest of the exit pupil on the camera chip. Only the pixels within this mask are applied for the analysis. The scaling function contains the normalized long-term illumination of the pupil which is unity for all pixels within the mask in the ideal 
case. In the non-ideal case, this long-term illumination is inhomogeneous to some extent due to contamination of the optics and non-equal camera pixel gain. The scaling function is applied to compensate this effect. An example image of pupil illumination is given in Fig. 3 (left). The speckle structure of intensity is clearly visible. Fig. 3, right, shows the corresponding pupil area as identified by the masking algorithm.
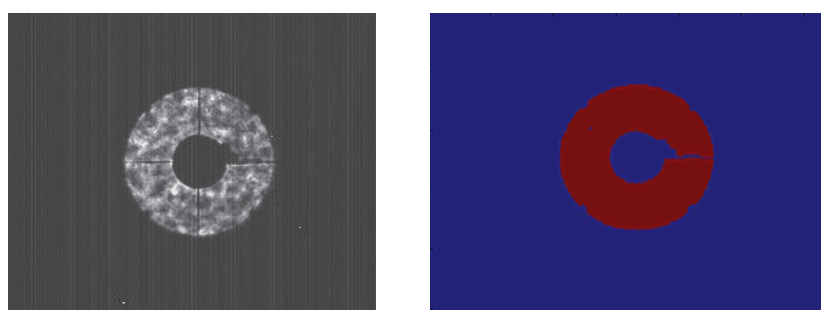

Fig. 3. Left: pupil image of intensity. Right: pupil area as identified by the masking algorithm.

The actual processing covers calculation of the covariance function from which the scintillation index and the correlation width are derived. In the first step, the 2-dimensional covariance function of intensity is calculated for each image. Next, the 1-dimensional covariance function is obtained by circular averaging. This is possible under the assumption of homogeneity and isotropy of turbulence. Multiple samples of the function are averaged (40 samples are used in the current analysis) to obtain one representation of the intensity covariance function. Eventually, scintillation index and correlation width are derived.

$I_{\text {meas }}$ contains the measurements of the spatial intensity field taken by one camera shot. This sample image is multiplied with the mask image and the scaling image to define the area of interest and correct the inhomogeneity of illumination:

$I_{\text {valid }}(x, y)=I_{\text {meas }}(x, y) \cdot M(x, y) \cdot S(x, y)$.

In order to remove the bias in the correlation function produced by the finite measurement area, i.e. the mask, the 2-dim autocorrelation function $A_{\text {valid }}(m, n)$ of the sample image is divided by the 2-dim autocorrelation function of the mask $A_{\mathrm{M}}(m, n)$. The variables $m$ and $n$ denote the spatial separation in the two directions. This results in the 2-dim unbiased autocorrelation function

$$
A_{\text {unbiased }}(m, n)=\frac{A_{\text {valid }}(m, n)}{A_{\mathrm{M}}(m, n)} \text {. }
$$

$A_{\text {unbiased }}(m, n)$ is averaged in circular direction to obtain the 1$\operatorname{dim}$ autocorrelation function $C(\rho)$ where $\rho[\mathrm{m}]$ is the spatial separation. Averaging over a set of samples results in the mean autocorrelation function $C_{\text {mean }}(\rho)$. The normalized covariance function $B(\rho)$ is calculated as

$$
B(\rho)=\frac{C_{\text {mean }}(\rho)}{I_{\text {mean }}^{2}}-1
$$

where $I_{\text {mean }}$ denotes the mean intensity of the sample images. Eventually, the covariance function value at $\rho=0$ gives the scintillation index, the $1 / \mathrm{e}$-drop of $B(\rho)$ gives the correlation width of intensity $\rho_{\mathrm{c}}[\mathrm{m}]$.

\section{RESULTS}

Pupil images were recorded in seven experiments, all during night-time. Table 2 gives a list of the measurement dates, times, experiment designation and exposure time. The local time was Central European Summer Time (UTC $+2 \mathrm{~h}$ ). The exposure time was always kept low enough to avoid time averaging of scintillation and high enough to achieve useful signal strength. However, the images of 2009 were noisy in some situations and thus, parts of the recording were discarded.

TABLE II. LIST OF ANALYSED EXPERIMENTS. THE DESIGNATION CONTAINS THE YEAR OF MEASUREMENT AND SEQUENTIAL NUMBERING OF THE TWO CAMPAIGNS. THE TIME CORRESPONDS TO $1^{\circ}$ POINTING ELEVATION

\begin{tabular}{|l|l|l|l|}
\hline \multicolumn{1}{|c|}{ Date } & \multicolumn{1}{|c|}{ Time [UTC] } & \multicolumn{1}{c|}{$\begin{array}{c}\text { Exp. } \\
\text { designation }\end{array}$} & $\begin{array}{c}\text { Exp. Time } \\
{[\boldsymbol{\mu s}]}\end{array}$ \\
\hline 9th June 2006 & $00: 00: 24$ & KT06-02 & 150 \\
\hline 14th June 2006 & $01: 02: 51$ & KT06-03 & 80 \\
\hline 15th June 2006 & $23: 52: 10$ & KT06-04 & 80 \\
\hline 28th June 2006 & $00: 46: 05$ & KT06-07 & 100 \\
\hline 1st July 2009 & $01: 28: 12$ & KT09-03 & 1000 \\
\hline 19th August 2009 & $02: 20: 34$ & KT09-05 & 100 \\
\hline 28th August 2009 & $02: 04: 38$ & KT09-08 & 100 \\
\hline
\end{tabular}

The measurement results for scintillation index and correlation width for the individual experiments are given in and Fig. 5.

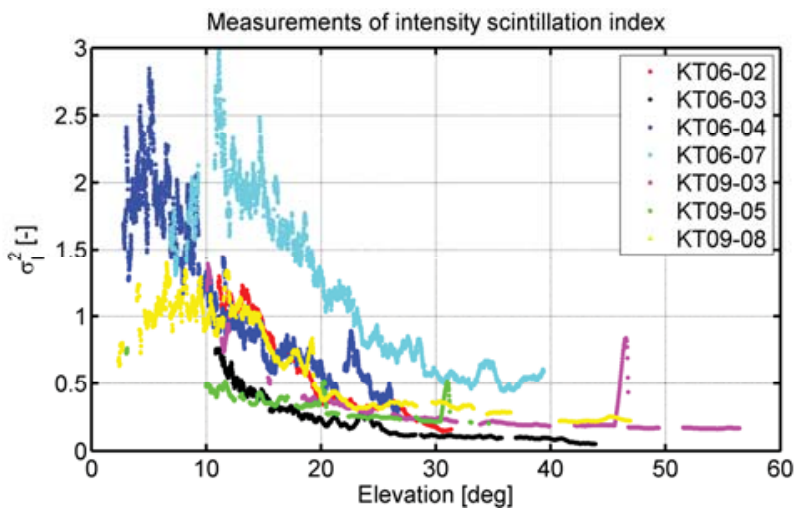

Fig. 4. Measurements of intensity scintillation index of LEO-ground downlink at $847 \mathrm{~nm}$ during KIODO Trials (KT) in 2006 and 2009. The seven lines denote individual experiments at different days. Measurements were taken during night time.

The run of the scintillation index shows clear saturation between 5 and 12 deg elevation angle and strongly decreasing values for high elevations. The values range from about 0.1 at high elevations to values of up to 3 at the scintillation peak. Also the correlation width shows clear saturation. Here values range from below $1 \mathrm{~cm}$ at high elevations to over $8 \mathrm{~cm}$ at the peak. The values of correlation width in KT09-05 as well as the values of KT09-03 and KT09-08 at high elevations are unrealistically low. Since in these measurements the signal was rather weak, camera noise is likely to influence the analysis and therefore cause the low values. These values are discarded for the further creation of weighted averages and mean values. The first are created by averaging a non-identical number of 
measurement samples within a 1-grid resolution of the elevation vector. The second is the mean of the weighted averages of all measurements.

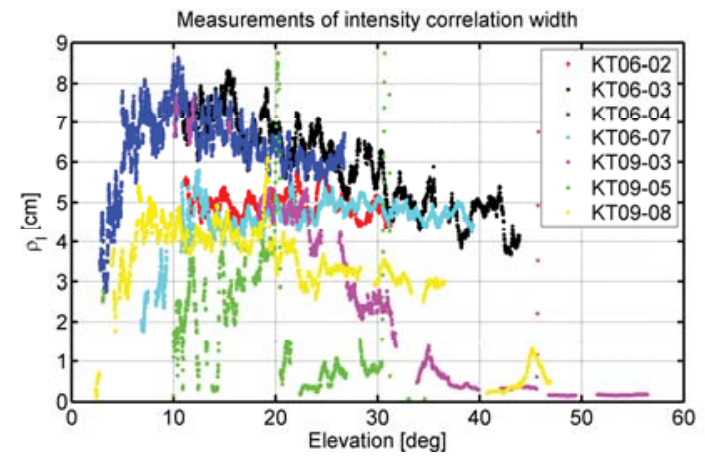

Fig. 5. Measurements of intensity correlation width of LEO-ground downlink at $847 \mathrm{~nm}$ during KIODO Trials (KT) in 2006 and 2009. The seven lines denote individual experiments at different days. Measurements were taken during night time.

The weighted averages of scintillation index and correlation width are shown in Fig. 6 and Fig. 7. The averages are created with an integral elevation grid thus smoothing the individual measurements.

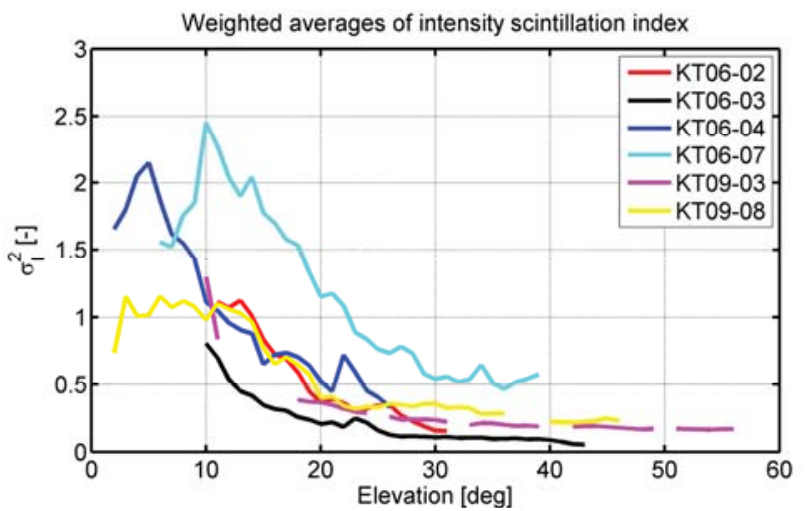

Fig. 6. Weighted averages of intensity scintillation index in excluding KT0905 .

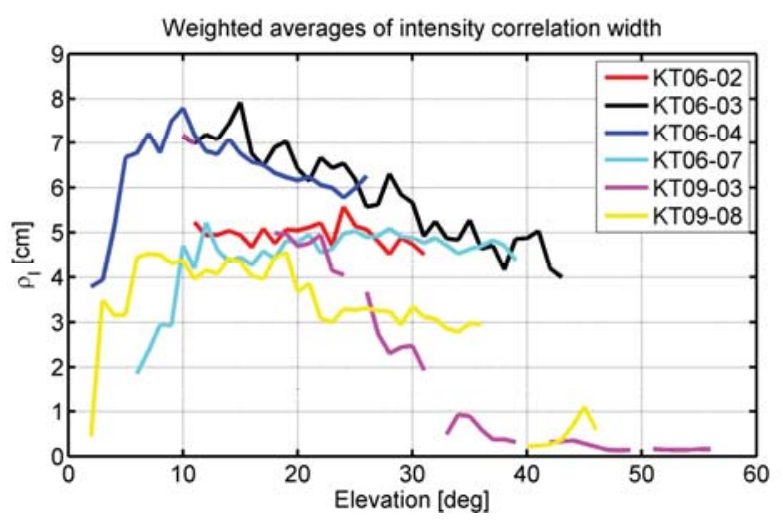

Fig. 7. Weighted averages of intensity correlation width in Fig. 5 excluding KT0

Fig. 8 and Fig. 9 contain the mean values of scintillation index and correlation width together with a model calculation. Both measurement curves match the theoretical curves fairly well. However, it must be noted that the number of measurements is rather limited and their spread is significant which suggests the need for more measurements, i.e. a larger data base.

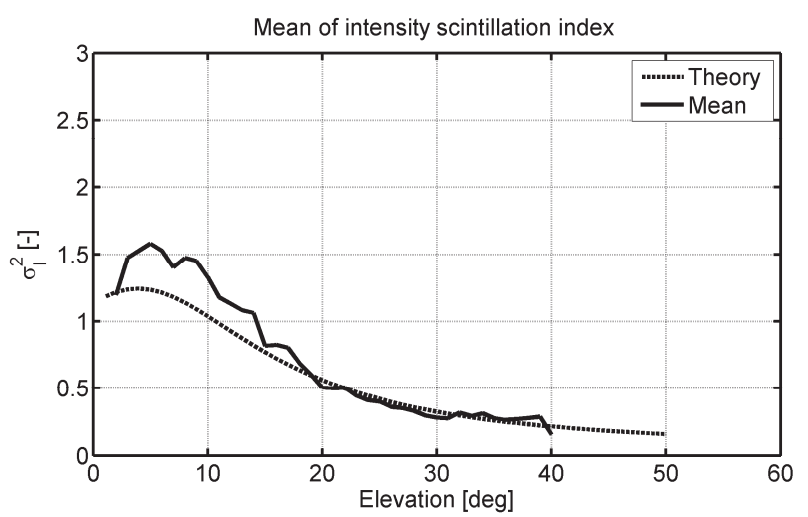

Fig. 8. Mean of intensity scintillation index from Fig. 6 and theoretical curve.

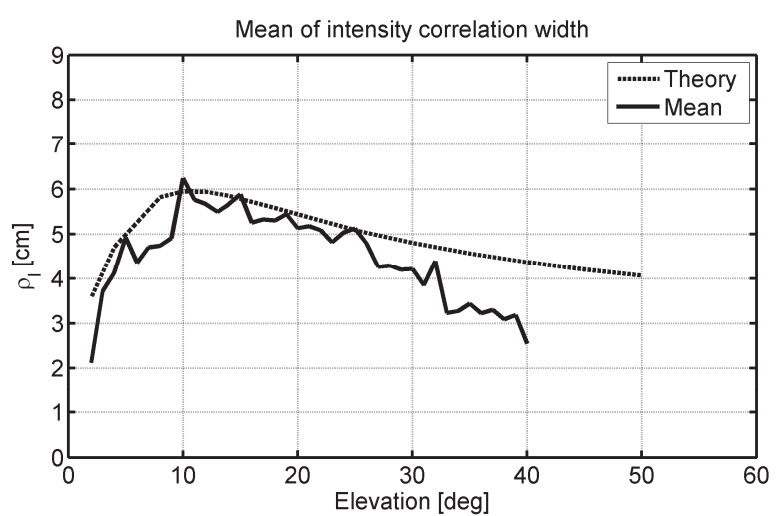

Fig. 9. Mean of intensity correlation width from Fig. 7 and theoretical curve.

However, the scintillation index deviates in the focusing regime from the model predictions. Inner scale effects are likely to be the reason since these are not covered by the theoretical model. No significant deviation of the theoretical curve from the correlation width measurements can be observed. Just at the higher elevation, above $30 \mathrm{deg}$, the curves deviate. The reason here may be the rather low values of KT09-03 and KT09-08 (Fig. 7) which might be due to the particular atmospheric conditions.

\section{CONCLUSION}

A preliminary analysis of recorded pupil images from satellite downlinks is presented. To some extent, the measurements agree well with the described theory, to some extent deviations are observed. The reasons might be non-ideal choice of the $C_{\mathrm{n}}{ }^{2}$-profile for modelling and/or insufficient description of the instantaneous turbulence effects by the used theoretical model. E.g. the compared model is only true for a Kolmogorov spectrum with its zero inner and infinite outer -5.cale which can have reasonable influence on the scintillation index. For better understanding of the measurements and the channel behavior, the analysis is going to be extended and further measurements obtained. In particular, an optimal use of low pass filters to suppress camera noise and frame averaging must be investigated. Furthermore, the pupil camera will be 
used to emulate different aperture sizes to quantify aperture averaging in the LEO-downlink.

\section{ACKNOWLEDGMENT}

The author would like to thank the all members of the DLR Institute of Communications and Navigation who participated in the KIODO campaign and contributed to the valuable measurement recordings. Special thanks go to JAXA and NICT who operated the OICETS satellite and the LUCE laser terminal.

\section{REFERENCES}

[1] V. I. Tatarski, The effects of the turbulent atmosphere on wave propagation. Israel Program for Scientific Translation, 1971.

[2] R. R. Beland, Atmospheric propagation of radiation - the infrared and electro-optical systems handbook, vol. 2. Bellingham: SPIE Press, 1996.
[3] L. C. Andrews and R. L. Philips, Laser Beam Propagation through Random Media, 2nd ed. Belligham: SPIE Press, 2005.

[4] L. C. Andrews, R. L. Phillips, and C. Y. Hopen, "Scintillation model for a satellite communication link at large zenith angles," SPIE Optical Engineering, vol. 39, pp. 3272-3280, 2000.

[5] N. Perlot, M. Knapek, D. Giggenbach, J. Horwath, M. Brechtelsbauer, Y. Takayama, and T. Jono, "Results of the Optical Downlink Experiment KIODO from OICETS Satellite to Optical Ground Station Oberpfaffenhofen (OGS-OP)," in Free-Space Laser Communication Technologies XIX and Atmospheric Propagation of Electromagnetic Waves, 2007, vol. 6457, p. 645704.

[6] F. Moll and M. Knapek, "Free-space laser communications for satellite downlinks: Measurements of the atmospheric channel," in Proc. of 62nd International Astronautical Congress, 2011. 